\title{
Transmit Antenna Selection for UHF MIMO Linking
}

\author{
Marjan Baghaie A. ${ }^{\dagger \S}$, Ian McLoughlin ${ }^{\S}$, Philippa A. Martin ${ }^{\dagger}$, Kishore Mehrotra ${ }^{\S}$ and Desmond P. Taylor ${ }^{\dagger}$ \\ ${ }^{\dagger}$ Department of Electrical and Computer Engineering \\ University of Canterbury, Christchurch, New Zealand \\ Email: marjan.baghaie@ ieee.org, \{p.martin, taylor\}@elec.canterbury.ac.nz \\ ${ }^{\S}$ Group Research, Tait Electronics Ltd., Christchurch, New Zealand \\ Email: \{ian.mcloughlin, kishore.mehrotra\}@tait.co.nz
}

\begin{abstract}
The use of multiple-input multiple-output (MIMO) configurations in wireless systems is becoming increasingly popular, due to the potential capacity enhancing properties. The use of such configurations, requires deployment of multiple RF chains and results in an increase in cost and complexity. This paper focuses on transmitter design, where cost is often dominated by the need to utilise linearized power amplifiers. Thus, the effect of reducing the number of active transmit antennae through selection can yield a significant cost advantage. Three criteria for transmit antenna selection are evaluated and characterised through the use of real channel data in the UHF band. The criteria are based only on the channel gains and have relatively low complexity; thus are suitable for practical purposes. This study examines the performance of the tested selection algorithms under a variety of operating conditions. The paper also considers the issue of delayed switch time due to reverse-link communication latency.
\end{abstract}

\section{INTRODUCTION}

Multi-antenna technology has emerged as a method for enabling high-speed wireless communications. Such systems have been shown to achieve larger capacities and improved performance in comparison to their single antenna counterparts [1]-[4]. However, for practical applications, the cost and complexity of implementation is significant due to the number of RF chains required. This is in particular the case for transmitters where costly linearisation is often needed. These concerns have motivated researchers to develop methods of reducing implementation cost while maintaining the benefits of MIMO systems. One such technique is antenna subset selection, which seeks to utilise a subset of the available transmit and/or receive antennas.

Various antenna subset selection criteria have been discussed in the literature, including power and signal-to-noise (SNR) maximisation [5]-[7] (generally norm-based algorithms), maximisation of the ergodic capacity of the equivalent channel [8], [9], and minimisation of the average probability of error [10], [8], [12], [17]. Although norm-based algorithms do not always produce the best results, they are useful due to their low computational complexity and known statistics.

This paper considers transmit antenna selection, specifically for UHF band multi-antenna systems. The algorithms discussed in this paper, are calculated based on the channel gains only (i.e. they do not require knowledge of the channel statistics) and are considered relatively simple to implement in a practical system. The performance of several antenna selection algorithms are investigated on real channel measurements.

The remainder of this paper is organised as follows: In section II, a number of criteria for transmit antenna selection are discussed and their application is investigated through the use of real data. Section III outlines the evaluation methods used to determine algorithm performance, while section IV presents the results. The paper is concluded in section V.

\section{SELECTION METHODOLOGY}

The wireless system considered in this paper consists of $N_{t}$ transmit and $N_{r}$ receive antennas. Such a system can be represented by

$$
\boldsymbol{y}=\boldsymbol{H} \boldsymbol{x}+\boldsymbol{n}
$$

where $y$ and $x$ are the received and transmitted signal vectors, respectively. $\boldsymbol{H}$ is the channel response matrix, and $\boldsymbol{n}$ represents the additive white Gaussian noise (AWGN) vector. To study transmit antenna selection, we assume there are $K_{t}$ RF chains available at the transmitter, where $K_{t}<N_{t}$. The transmitter needs to select $K_{t}$ out of the $N_{t}$ available physical transmit antennas for data transmission. There is a total of $C_{K_{t}}^{N_{t}}$ possible combinations of transmit antennas that can be used for transmission. Transmission occurs for a fixed frame length (itself determined by channel stationarity or some other criterion), after which the transmitter selection process repeats. Note that in a practical system, one frame might be too short.

It is assumed that the receiver estimates the channel during a training period. The desired transmit antenna subset is then computed and the choice is fed back to the transmitter through a low bandwidth feedback path (this configuration is further explained in Section III). Since this message takes some time, the switching is not instantaneous, but occurs in time for the subsequent packet. It has been shown that in the absence of channel knowledge at the transmitter, the optimal solution is to distribute power equally across all $N_{t}$ available antennas [1], [2], [3], [13].

In this paper, we consider the following subset selection algorithms:

1) Power Criterion: In general, for a $\left(N_{r} \times N_{t}\right)$ channel matrix, the received SNR is proportional to the Frobe- 
nius norm of the selected $\left(N_{r} \times K_{t}\right)$ channel submatrix and can be represented by

$$
\boldsymbol{S N R}=\gamma_{s} \sum_{i=1}^{N_{r}} \sum_{j=1}^{K_{t}}\left|H_{i, j}\right|^{2}
$$

where $\gamma_{s}=\frac{E_{s}}{N_{0}}$ is the energy per transmit antenna divided by the noise power, and $H_{i, j}$ is the channel gain between the $j$ th transmit antenna (in the selected subset) and the $i$ th receive antenna.

In order to select the optimal combination of transmit antennas, this method tries to minimize the bit error rate (BER) by choosing the subset of antennae corresponding to the maximum SNR. This is equivalent to selecting those columns of $\boldsymbol{H}$ that maximize (2) [7], [14].

2) Full Conditioning: This implies choosing the subset of $K_{t}$ antennas corresponding to the best condition number from the channel matrix-derived submatrices. This condition was introduced as some of the channels selected by the power criterion tended to be ill-conditioned, leading to poor performance. This is likely to be innate to the nature of the long distance UHF channels under consideration.

3) Zero-Forcing Based Selection (ZFS): This method is primarily based on zero-forcing [15]. It assumes the total transmit power is constant but can be distributed nonuniformly across different antennas. The method works by choosing the subset of $K_{t}$ antennas that minimize

$$
\xi=\sum_{i=1}^{K_{t}} \sum_{j=1}^{N_{r}}\left|H_{i, j}^{\#}\right|^{2}
$$

where $\boldsymbol{H}^{\#}$ denotes the pseudo-inverse of the selected channel matrix, defined as $\boldsymbol{H}^{\#}=\left(\boldsymbol{H}^{H} \boldsymbol{H}\right)^{-1} \boldsymbol{H}^{H}$.

An advantage of this method is that by minimizing a matrix expression based on the pseudo-inverse of the channel, ill-conditioned channels are automatically disqualified from selection. Similar approaches to this have been described and analysed in the literature [11], [8], [16]. A derivation is outlined in the Appendix.

In this paper, the application of these approaches to UHF band MIMO systems are illustrated and evaluated using measured channel data.

\section{SYSTEM MODEL}

A custom-designed multi-channel phase-locked UHF transceiver testbed was used for the experiments. The testbed was optimized to explore channel characteristics in the absence of modulation effects, which can be evaluated as and when appropriate using system simulation tools. This process has been discussed in [4].

The testbed was set up to have an equal number of transmit and receive antennas, namely $N_{t}=N_{r}=4$, where $K_{t}=2$ of the $N_{t}=4$ transmit antennas were to be selected for transmission. The system operated at a frequency of 415 $\mathrm{MHz}$, with a $25 \mathrm{kHz}$ bandwidth. An unmodulated carrier was

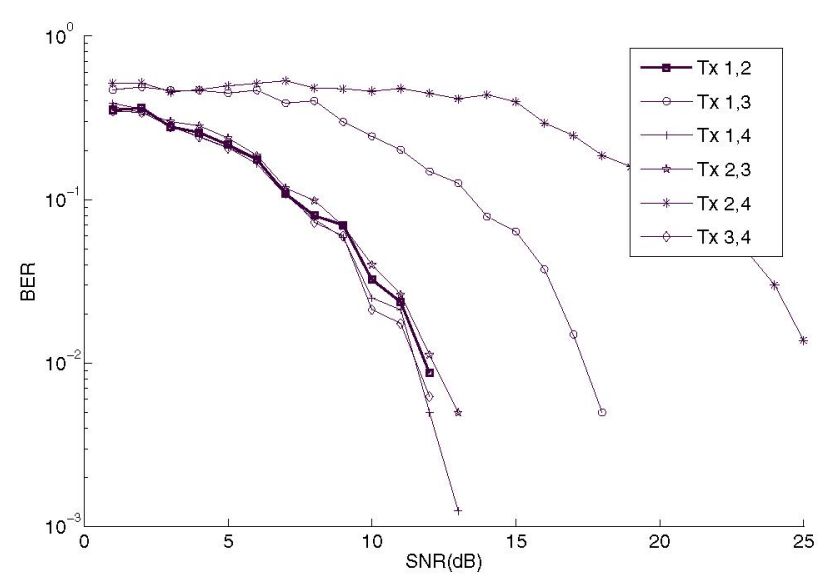

Fig. 1. Error performance for all possible transmit antenna combinations for one particular captured channel.

transmitted over each such channel in a rapid time slotted fashion (where all combinations of two transmit antennas were considered). The received signal was captured in four channels at distances of up to $30 \mathrm{~km}$ over various terrains.

Only minor obstructions were present along the investigated paths, with path losses of between 130 and $150 \mathrm{~dB}$ for 37 $\mathrm{dBm}$ or 5 watts of transmit power per antenna. Therefore, the receive power was between -93 and $-113 \mathrm{dBm}$. Assuming the $1 / r^{4}$ law for power loss vs distance this allowed a range of approximately $30 \mathrm{~km}$ between the transmitter and the receiver. Seven element Yagi antennas were used. To evaluate error performance, a 256-QAM constellation was superimposed on the detected and estimated channel matrices, and symbols were applied at a rate of approximately $20 \mathrm{k}$ symbols $/ \mathrm{sec}$.

\section{RESULTS}

Each of the three criteria described in Section II were used to select an optimal subset of antennas for several hundred captured channels. The results were then evaluated and compared based on two different performance measures, namely SNR and BER. For visualization purposes, only 50 channels are presented in the plots. The y-axis component has been scaled to fit in all cases.

As an example, system error performance is presented in Fig. 1 for one set of 4 by 4 captured channels, assuming equal power per transmit antenna. The BER curves are plotted for the 6 possible transmit antenna pairs that can be selected. As can be seen, two of the combinations, namely $(1,3)$ and $(2,4)$, yield error performance much worse than the other combinations. The power criterion selects antennas 1 and 2 as the optimal pair in this case.

\section{A. SNR Based Performance Measure ( $\xi$ )}

As shown in the Appendix, $\xi$ is inversely proportional to the SNR for any selection criterion. It can be used to provide a measure of comparison between different selection 


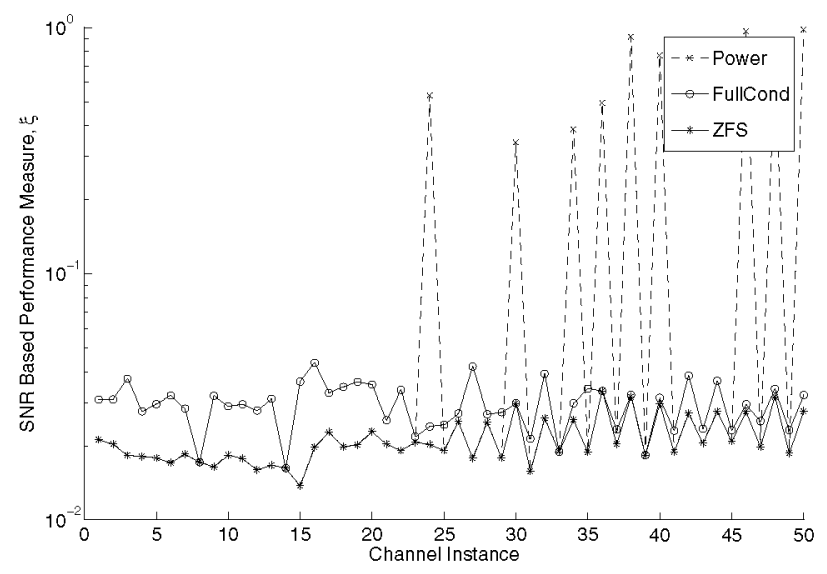

Fig. 2. Performance of various selection criteria in terms of $\xi$.

methodologies. Fig. 2 presents $\xi$ plotted for 50 samples of the captured channels selected by each of the selection methods.

As is evident in Fig. 2, the power criterion of (2) performs very poorly in some instants, leading to the spikes which indicate severe performance degradation. This is suspected to be due to the method not being able to deal with ill-conditioned channels. Full-conditioning does not have that problem but it is not as effective on average (in terms of SNR performance) as the power criteria. ZFS outperforms the other methods by taking both condition number and power into account.

The experiment was repeated for randomly computergenerated Rayleigh and Rician channels. In both cases the order of performance was similar to that observed in the captured channels but the performance difference of the various criteria was of lower significance. In the Rician case, this difference became larger as the Rician constant was increased.

\section{B. BER Based Performance Measure}

BER values were also determined for the different selection criteria (simulated for 256 QAM), and the results for an arbitrary SNR of $10 \mathrm{~dB}$ are presented in Fig. 3. Note that since we are plotting BER values, the lower the curve the better the corresponding performance.

As can be seen, the power criterion still faces problems, suspected to be due to the ill-conditioning of some captured channel matrices. ZFS is again seen to outperform the power criterion by taking both power and the channel condition into account. In contrast to the results obtained using $\xi$ however, the full-conditioning method now outperforms all the other methods. This is suspected to be due to the way the simulation was performed. That is, in order for the BER-SNR calculations to be performed in the simulation, the selected 4 by 2 channel matrix was normalized before being passed to the receiver (to give an equivalent channel power of unity). It can therefore be argued that the simulation was biased in favour of the full-conditioning method, where the normalization tends to be irrelevant to performance in the absence of signal rounding and quantization effects.

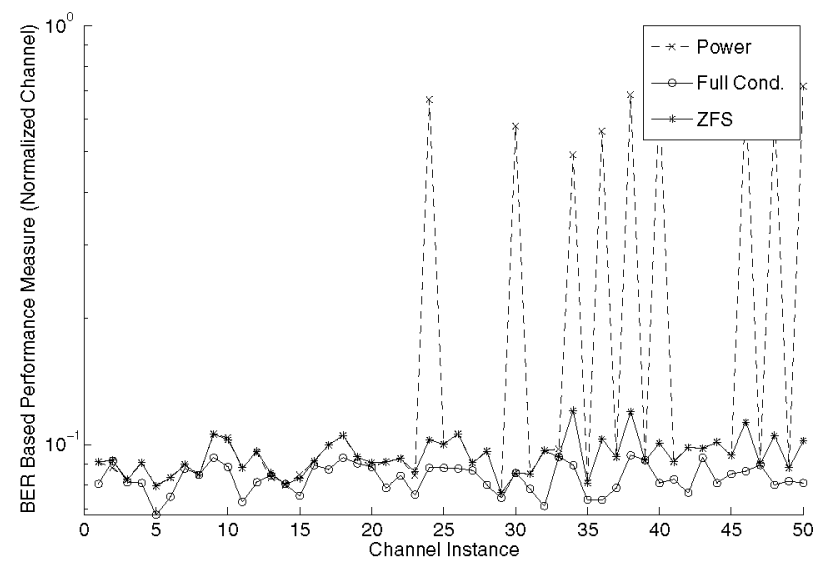

Fig. 3. BER performance for a SNR value of $10 \mathrm{~dB}$.

It is worth noting that this normalization process is commonly applied in published simulation results, where it is performed in order to set received SNR and measure the corresponding BER, yielding the standard BER versus SNR curve as presented in Fig. 1. In order to derive a more impartial representation of the performance of each method, the normalization factor used was plotted for each channel and the results are presented in Fig. 4.

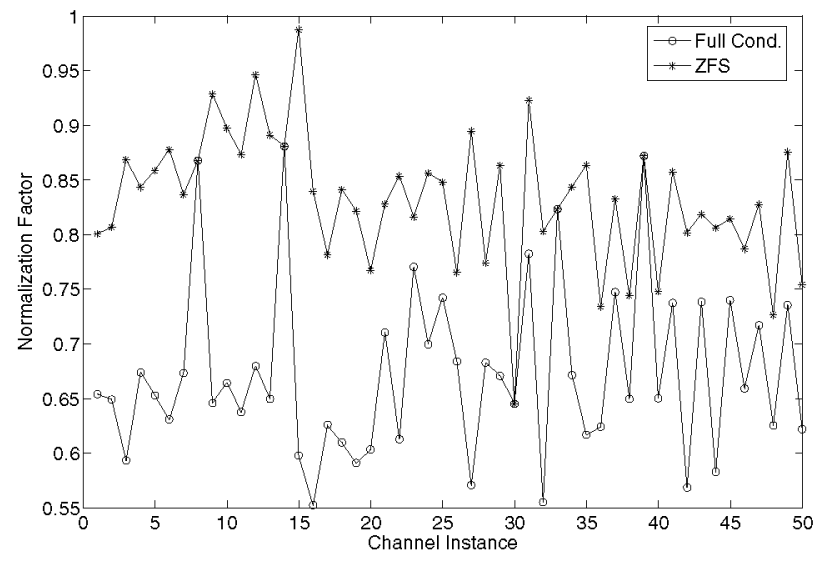

Fig. 4. Comparison of the normalization factor used in ZFS and full conditioning.

The normalization factor is directly proportional to the channel power, i.e. larger normalization factors represent better channels in terms of power. The result highlights the ineffectiveness of the full-conditioning method in terms of power. The power criterion, based on its definition, would clearly outperform the alternative methods under such conditions. As before, ZFS proves to be a good compromise (between the power and condition number criteria).

Thus, instead of calculating BER for a fixed SNR, Fig. 5 presents the BER values, evaluated this time for fixed noise 


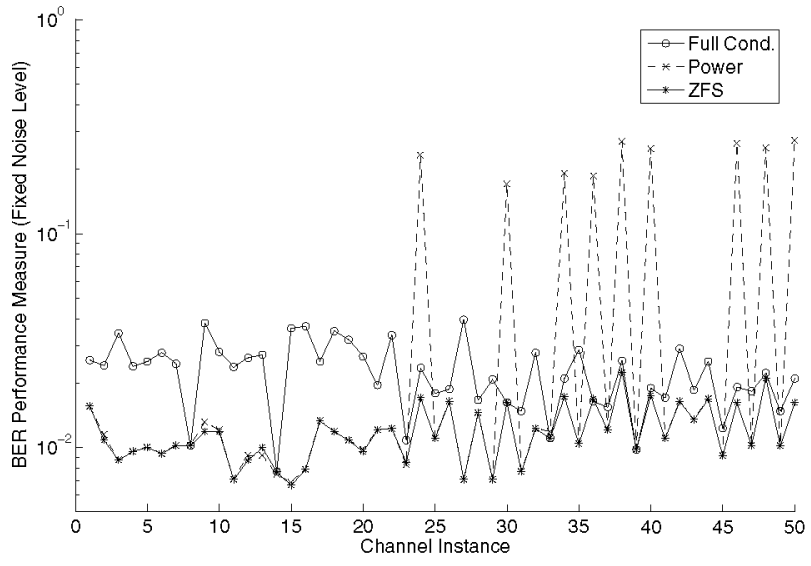

Fig. 5. BER based performance of selection criteria for an equivalent fixed noise level.

levels (without normalizing the channel). These results now agree with those obtained based on $\xi$, in Fig. 2.

\section{Feedback Consideration:}

During the initial experiments, it was assumed that the selected channel information was relayed back to the transmitter via a feedback path of sufficient speed to indicate a choice of antenna subset before the end of synchronisation and training. Such switching was effectively instantaneous, as far as data packets were concerned. However, this is unlikely to be true for most deployed systems, where the feedback is likely to comprise part of a messaging protocol; and is thus delayed to fit in with formatting, framing and packetisation. In simulations, the effect of a delay due to the feedback path was modeled by simulation to incorporate a fixed one packet delay between antenna subset selection and the subsequent switch. It was found that results for all three cases were slightly worse, however, their relative order of performance stayed the same.

\section{CONCLUSION}

Three transmit antenna subset selection criteria were investigated to consider their practicability for use in long distance UHF MIMO channels using a 256-QAM modulation format. These criteria were power, full conditioning and the ZFS method. The power criteria outperforms full conditioning in well-conditioned channels, but leads to extremely poor performance when the channel is ill-conditioned. Full conditioning eliminates the effect of ill-conditioned channels. ZFS outperforms all other investigated methods in terms of SNR performance. It takes the channel power into account, and by minimizing the pseudo-inverse of the channel, automatically eliminates the problems of ill-conditioned channels. The cost is a slightly higher degree of computational complexity. When channel state information (CSI) is available at the transmitter, the ZFS discussed can also be used to distribute the transmit power optimally (in a zero-forcing sense) to different antennas.

\section{APPENDIX I \\ DERIVATION OF ZFS CRITERION}

This method relies upon the channel (pseudo) inverse and is derived from the zero-forcing (ZF) equations [15]. Similar approaches to this have been described and analysed in the literature [11], [8], [16]. This paper uses a slight variation of these methods and explores their application to UHF band multi-antenna systems.

Consider the $\left(N_{r} \times N_{t}\right)$ MIMO system described in (1). Assume there exists only $K_{t}\left(<N_{t}\right)$ RF chains at the transmitter, from which the data can be transmitted. This would mean that the $\left(N_{r} \times N_{t}\right)$ system reduces to a $\left(N_{r} \times K_{t}\right)$ system and the goal is to choose the best $K_{t}$ antennas out of the available $N_{t}$. It is further assumed that the ZF method is used for decoding.

Considering independent noise, the ZF data estimate, for a given subset selection is

$$
\widehat{\boldsymbol{x}}=\left(\boldsymbol{H}^{H} \boldsymbol{H}\right)^{-1} \boldsymbol{H}^{H} \boldsymbol{y}=\boldsymbol{H}^{\#} \boldsymbol{y}
$$

We can then write

$$
\widehat{\boldsymbol{x}}=\boldsymbol{x}+\boldsymbol{H}^{\#} \boldsymbol{n}
$$

and it follows that the $i$ th component has the form

$$
\widehat{x_{i}}=x_{i}+\sum_{j=1}^{N_{r}} H_{i, j}^{\#} n_{j}
$$

Assuming that the noise is independent, we then obtain a measure of SNR for the system given the $K_{t}$ transmit antennas as

$$
S N R_{\text {total }}=\sum_{i=1}^{K_{t}} S N R_{i}=\sum_{i=1}^{K_{t}} \frac{\sigma_{x_{i}}{ }^{2}}{\sigma_{n}{ }^{2} \sum_{j=1}^{N_{r}}\left|H_{i, j}{ }^{\#}\right|^{2}}
$$

where $\sigma_{x}^{2}$ and $\sigma_{n}^{2}$ represent the signal and the noise power, respectively.

For optimum (SNR-based) performance, it is desirable to maximize $S N R_{\text {total }}$ for a given signal and noise power. Therefore, the goal is to maximize $S N R_{\text {total }}$ given constant $\sigma_{x_{\text {total }}}{ }^{2}$ and ${\sigma_{n}}^{2}$, where

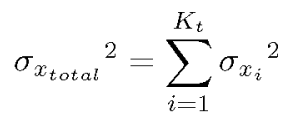

In a practical system, one can assume that the overall channel gain is less than unity (i.e. no extra signal energy gained during transmission). Therefore, it can be assumed that

$$
\sum_{j=1}^{N_{r}}\left|H_{i, j} \#\right|^{2}>1, \quad i=1, \ldots, K_{t}
$$

We can now consider two cases: 


\section{A. Equal Power Distribution:}

For an equal power distributer

$$
{\sigma_{x_{i}}}^{2}=\frac{{\sigma_{x_{\text {total }}}}^{2}}{K_{t}}, \quad i=1, \ldots, K_{t}
$$

and (7) can be simplified to

$$
S N R_{\text {total }}=\frac{\sigma_{x_{\text {total }}{ }^{2}}}{K_{t} \sigma_{n}{ }^{2}} \times \sum_{i=1}^{K_{t}} \frac{1}{\sum_{j=1}^{N_{r}}\left|H_{i, j}\right|^{2}}
$$

Considering constant signal and noise power and also (9), it is clear that in order to maximize (11), we need to minimize the denominator. Therefore, we need to choose the rows of $\mathbf{H}^{\#}$ which lead to a minimum absolute row squared sum (i.e. the criterion highlighted in (3)).

\section{B. Non-equal Power Distribution:}

For a non-equal power distributer, the optimum distribution (assuming fixed total power) should be such that

$$
\frac{\sigma_{x_{i}}{ }^{2}}{\sum_{j=1}^{N_{r}}\left|H_{i, j}{ }^{\#}\right|^{2}}=\frac{\sigma_{x_{z}}{ }^{2}}{\sum_{j=1}^{N_{r}}\left|H_{z, j}{ }^{\#}\right|^{2}}, \quad z, i=1, \ldots, K_{t}
$$

Therefore,

$$
\sigma_{x_{z}}{ }^{2}=\sigma_{x_{i}} \frac{\sum_{j=1}^{N_{r}}\left|H_{z, j} \#\right|^{2}}{\sum_{j=1}^{N_{r}}\left|H_{i, j} \#\right|^{2}}
$$

Considering (13) and (8), for $i=1, \ldots, K_{t}$, we can write

$$
\begin{aligned}
\sigma_{x_{\text {total }}}{ }^{2}=\sigma_{x_{i}}{ }^{2}+\sum_{\substack{z=1 \\
z \neq i \\
z \neq i}}^{K_{t}} \sigma_{x_{z}}{ }^{2} \\
=\sigma_{x_{i}}{ }^{2}\left(1+\sum_{\substack{z=1 \\
z \neq i}}^{K_{t}} \frac{\left.\sum_{j=1}^{N_{r}}\left|H_{z, j} \#\right|^{2}\left|H_{i, j} \#\right|^{2}\right)}{N_{r}}\right.
\end{aligned}
$$

Using (14) and (7), for $i=1, \ldots, K_{t}$

$$
\begin{gathered}
S N R_{i}=\frac{\sigma_{x_{\text {total }}{ }^{2}}}{\sigma_{n}{ }^{2}\left(\sum_{j=1}^{N_{r}}\left|H_{i, j} \#\right|^{2}\right)\left(1+\sum_{\substack{z=1 \\
z \neq i}}^{K_{t}} \frac{\sum_{j=1}^{N_{r}}\left|H_{z, j} \#\right|^{2}}{\sum_{j=1}^{N_{r}}\left|H_{i, j} \#\right|^{2}}\right)} \\
=\frac{\sigma_{x_{\text {total }}{ }^{2}}}{\sigma_{n}{ }^{2} \sum_{z=1}^{K_{t}} \sum_{j=1}^{N_{r}}\left|H_{z, j} \#\right|^{2}}
\end{gathered}
$$

Assuming that the total signal power and noise power are constants, it is evident that in order to maximize (15) and therefore (7), we need to select the subset of $K_{t}$ rows which minimize

$$
\xi=\sum_{z=1}^{K_{t}} \sum_{j=1}^{N_{r}}\left|H_{z, j} \#\right|^{2}
$$

leading to the criterion presented in (3). $\xi$ can be used as a performance measure for any of the considered selection criteria.

\section{REFERENCES}

[1] E. Telatar, "Capacity of Multi-Antenna Gaussian Channels," AT\&TBell Labs Internal Tech. Memo., June 1995

[2] G. J. Foschini, Jr., M. J. Gans, "On Limits of Wireless Communication in a Fading Environment When Using Multiple Antennas," Wireless Pers. Commun., Vol. 6, no. 2, pp. 311-335, March 1998

[3] J. Winters, "The Impact of Antenna Diversity on the Capacity of Wireless Communication Systems," IEEE Trans. Commun., vol. 42, pp. 1740-51, Feb. 1994

[4] M. Baghaie A., S. Kuo, I. V. McLoughlin, "FPGA Implementation of Space-Time Block Coding Systems," In Proc. IEEE 6th CAS Symp Front. Of Mobile and Wireless Commun., Vol. 2, pp. 591-94, June 2004

[5] S. Sanayei, A. Nostatinia, "Asymptotic Capacity Analysis of Transmit Antenna Selection," In Proc. ISIT, pp. 241, July 2004

[6] A. F. Molisch, M. Z. Win, J. H. Winters, "Capacity of MIMO Systems With Antenna Selection," In Proc. ICC, Vol. 2, pp. 570-574, June 2001

[7] D. Gore, A. Paulraj, "Space-Time Block Coding with Optimal Antenna Selection," In Proc. ICASSP, vol.4, pp. 2441 2444, May 2001

[8] A. D. Gore, R. W. Heath Jr., A. J. Paulraj, "Transmit Selection in Spatial Multiplexing Systems," IEEE Commun. Lett., Vol. 6, Issue 11, pp. 491493, Nov. 2002

[9] D. Gore, R. U. Nabar, and A. J. Paulraj, "Selecting an Optimal Set of Transmit Antennas for a Low Rank Matrix Channel," In Proc. ICASSP, Vol. 5, pp. 2785-2788, Istanbul, Turkey, June 2000

[10] D. Gore, A. Paulraj, "Statistical MIMO Antenna Sub-set Selection with Space-time Coding," In Proc. ICC, Vol. 1, pp. 641-645, May 2002

[11] R. W. Heath, Jr., S. Sandu, A. Paulraj, "Antenna Selection for Spatial Multiplexing Systems with Linear Receivers," IEEE Commun. Lett., Vol. 5, Issue 4, pp. 142 - 144, April 2001

[12] A. Gorokhov, "Antenna Selection Algorithms for MEA Transmission Systems," In Proc. ICASSP, Vol. 3, pp. III-2857 - III-2860, May 2002

[13] T. Cover, J. Thomas, Elements of Information Theory, John Wiley and Sons, New York, 1991

[14] M. Torabi, M. R. Soleymani, "Antenna Selection for Turbo Coded MIMO OFDM Systems," 22nd Biennial Symp. Commun., Dept. Elec. and Computer Eng, Queen's Uni., Ontario, Canada, June 2004

[15] J. G. Proakis, Digital Communications, 4th ed., McGraw-Hill, 2001

[16] R. W. Heath Jr., D. J. Love, "Dual-mode antenna selection for spatial multiplexing systems with linear receivers", In Proc. 37th Asilomar Conf. on Signals, Systems and Computers, Vol. 1, pg. 1085 - 1089, Nov. 2003

[17] A. Gorokhov, D. Gore, A. Paulraj, "Performance Bounds for Antenna Selection in MIMO Systems," In Proc. ICC, Vol. 5, pp. 3021-3025, May 2003 